\title{
Parásitos del pez luna Mola mola (Pisces: Molidae). Primer registro en aguas de la costa centro sur de Chile
}

\section{Parasites of oceanic sunfish Mola mola (Pisces: Molidae). First record in waters from south-central Chile coast}

\author{
Italo Fernández ${ }^{1 *}$, Ciro Oyarzún², Ariel Valenzuela², Carolina Burgos², Víctor Guaquín² \& Víctor \\ CAMPOS $^{1}$ \\ 1Departamento de Microbiología, Facultad de Ciencias Biológicas Universidad de Concepción, Concepción, Chile \\ 2Laboratorio de Piscicultura y Patología Acuática, Facultad de Ciencias Naturales y Oceanográficas Universidad de \\ Concepción, Concepción, Chile \\ *itfernan@udec.cl
}

\begin{abstract}
RESUMEN
Este estudio reporta los parásitos presentes en un ejemplar de Mola mola, encontrado en la costa de Tomé, Chile, durante el mes de octubre de 2014. Se aislaron estados adultos de los digeneos Accacladium serpentulum y Accacladocoelium macrocotyle, del cestodo Anchistrocephalus microcephalus y del copépodo Cecrops latreillii. Además, se identificaron larvas de tercer estado del nematodo Anisakis sp. Tipo I, sensu Berland, 1961 (tercer estadio, L3) y plerocerci del cestodo Molicola horridus. Los resultados demuestran la participación de M. mola como hospedero definitivo, intermediario o paraténico en los ciclos de vida de los parásitos en el presente estudio.
\end{abstract}

Palabras clave: Parasitismo, Ciclos de vida, Patología.

\begin{abstract}
In this study, we report parasitological findings in one Mola mola specimen examined and collected as by catch-off Tomé, south central Chile, during october 2014. Adult stages of digeneans, Accacladium serpentulum and Accacladocoelium macrocotyle, cestode Anchistrocephalus microcephalus, and copepod Cecrops latreillii were isolated. Besides, at the larval stage, the third larval stage (L3) of nematode Anisakis sp. Type I, sensu Berland, 1961, and plerocerci larvae of cestode Molicola horridus were also observed. The results demonstrated the participation of M. mola as a definitive and intermediate or paratenic host in the life cycles of different parasites identified in the present study.
\end{abstract}

KeYwords: Parasitism, Life Cycles, Pathology.

La Familia Molidae comprende especies de peces epipelágicos, conocidos comúnmente como molas o peces luna. De acuerdo a Parenti (2003), esta familia está compuesta por cuatro especies: Mola mola (Linnaeus, 1758), Mola ramsayi (Giglioli, 1883), Ranzania laevis (Pennant, 1776) y Masturus lanceolatus (Liénard, 1840). Sin embargo, existen algunas controversias sobre la taxonomía de este grupo. Por ejemplo, estudios más recientes indican que, en aguas de Japón, existirían hasta tres especies diferentes, solo del género Mola (Yoshita et al. 2009). Las molas presentan distribución global aunque se les encuentra preferentemente en aguas cálidas y tropicales (Houghton et al. 2006; Pope et al. 2010). En Chile, se ha reportado ocasionalmente la presencia de $M$. lanceolatus, $R$. laevis y $M$. mola principalmente en aguas del norte del país, siendo $M$. ramsayi la especie más frecuentemente informada en aguas de mayor latitud, lo que coincidiría con su área preferente de presentación limitada al hemisferio sur (Pequeño 1989, 1997; Bass et al. 2005). Más allá de esta tendencia, la ocurrencia de mólidos en aguas de Chile es poco frecuente y estaría ligada a migraciones ocasionales influenciadas por cambios oceánicos atmosféricos producidos por el evento de El Niño (Sielfeld et al. 2010).

Debido a su extensa distribución, los peces de esta familia se constituyen en hospederos de una gran variedad de organismos parásitos. Sin embargo, los estudios que abordan la conformación de su fauna parasitaria son escasos (Ahuir- 
Baraja 2012), ya que al no constituir un recurso pesquero comercial, la información disponible deriva principalmente de la pesca incidental o del análisis de ejemplares varados en playas, muertos o debilitados, lo que determina informes esporádicos y escasez de ejemplares con los que efectuar estudios detallados. En aguas de Chile la información disponible sobre la parasitofauna de esta familia de peces es escasa y dispersa. Hasta la fecha, solo existen reportes parasitarios en M. ramsayi capturados en forma incidental (Atria 1967, 1977; Villalba \& Fernández 1985). Además, en un ejemplar de M. mola, varada en la costa de Valparaíso, se reportó la presencia del copépodo Lepeophtheirus nordmanni (Milne Edwards, 1840) (Stuardo 1958; Muñoz \& Olmos 2007).

Con el fin de aportar información respecto de la composición taxonómica de parásitos en peces $M$. mola y contribuir al conocimiento biológico de estos animales, consideramos de interés informar sobre el hallazgo de estos organismos en un ejemplar que se encontró en la costa centro sur de Chile.

En octubre de 2014, un ejemplar de M. mola fue encontrado varado y muerto en las cercanías de la costa de Tomé $\left(36^{\circ} 37^{\prime} 08^{\prime \prime}\right.$ S; $\left.72^{\circ} 57^{\prime} 47^{\prime \prime} \mathrm{W}\right)$ por el Servicio Nacional de Pesca de Chile, entidad que los remitió al Laboratorio de Piscicultura y Patología Acuática de la Facultad de Ciencias Naturales de la Universidad de Concepción (UdeC). La determinación de la especie se hizo en base a las características morfológicas definidas por Fraser-Brunner (1951). Se determinó el sexo, estado de madurez, longitud y peso del ejemplar (Tabla 1). Se realizó una exhaustiva inspección externa tras la cual se efectuó la necropsia al espécimen, que reveló la presencia de numerosos ejemplares parásitos, los que fueron visualizados en su mayoría en el tracto gastrointestinal y en las vísceras. Para facilitar el análisis de la ubicación de los parásitos dentro del tubo digestivo, se ató los extremos esofágico y anal para evitar la pérdida de su contenido y tres segmentos correspondientes a los tercios anterior, medio y posterior del intestino. El contenido intestinal y las vísceras fueron extraídas y se tamizaron, por separado, con suero fisiológico a presión en un cilindro plástico cuyo fondo contenía una malla de $0.50 \mathrm{~mm}$ de luz. El material retenido por los tamices fue examinado mediante lupa estereoscópica (Zeiss Stemi DRC, aumento 4x), lo que permitió separar el contenido alimentario y los ejemplares parásitos, en el caso del análisis del contenido intestinal. Los parásitos colectados fueron fijados en alcohol al 70 $\% \mathrm{y}$, con el fin de determinarlos taxonómicamente, fueron llevados al Laboratorio de Parasitología, de la Facultad de Ciencias Biológicas de UdeC. Los ejemplares de nematodos y digeneos fueron diafanizados con Lactofenol de Amman para visualizar sus estructuras internas. La identificación se efectuó mediante el uso de microscopía óptica (Motic, BA $310,10 x$ y 40x) y literatura especializada de referencia. Para la determinación taxonómica de los digeneos se consultó a Bray \& Gibson (1977); para los nematodos a Yamaguti (1961) y Berland (1961); para los cestodos a Bray et al. (1994) y para los copépodos a Grabda (1973).

TABLA 1. Lugar de rescate, características reproductivas y biométricas, y parásitos encontrados en un pez Mola mola del centro sur de Chile analizado en el presente estudio.

TABLE 1. Location rescue, reproductive and biometrical characteristics, and parasites found of one sunfish Mola mola from south-central Chile analyzed in the present study.

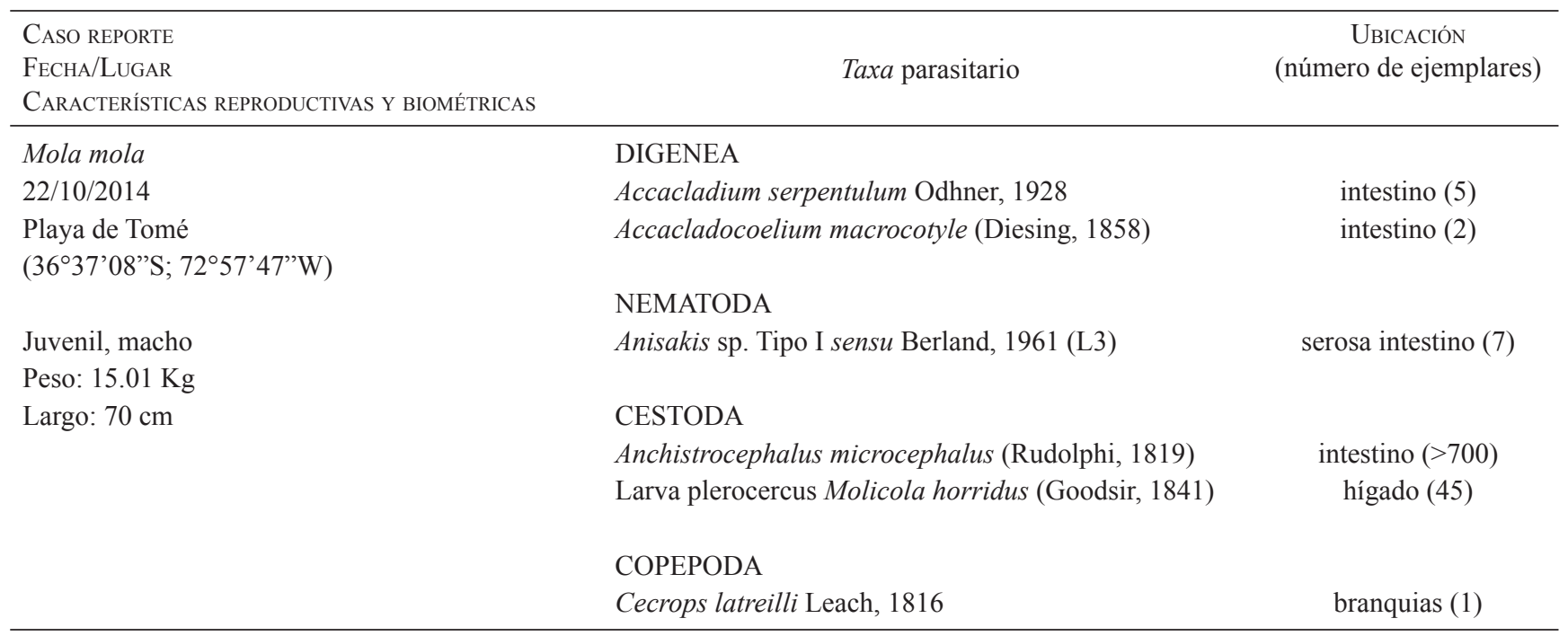


Se determinaron seis taxa parasitarios. Al estado adulto se identificó a los digeneos Accacladium serpentulum Odhner, 1928 y Accacladocoelium macrocotyle (Diesing, 1858), el cestodo Anchistrocephalus microcephalus (Rudolphi, 1819) y al copépodo Cecrops latreillii Leach, 1816. Al estado larval se encontró la larva de tercer estado del nematodo Anisakis sp. Tipo I sensu Berland, 1961 y larvas plerocerci del cestodo Molicola horridus (Goodsir, 1841). La determinación taxonómica, ubicación anatómica y cantidad de los parásitos encontrados en el ejemplar de $M$. mola en cuestión se detalla en la Tabla 1. La mayoría de los taxa parasitarios, al estado adulto, se ubicaron en el tracto gastrointestinal. El análisis de su ubicación anatómica reveló que en su distribución no existieron co-ocurrencias. En el estómago y dos tercios anteriores del intestino se presentó el cestodo A. microcephalus mientras que los digeneos $A$. serpentulum y $A$. macrocotyle se alojaron preferentemente en el intestino posterior. Solo se encontró un ejemplar macho del copépodo C. latreilli adosado laxamente al arco branquial derecho. Las formas larvales se concentraron en la serosa intestinal (Anisakis sp. Tipo I, L3) e hígado (plerocerci de $M$. horridus). Cabe destacar que se encontró, aproximadamente, 700 ejemplares adultos del cestodo $A$. microcephalus. Por último, una lista taxonómica de los parásitos encontrados en peces de la familia Molidae en aguas de Chile, incluidos los reportes del presente estudio, se indica en la Tabla 2.

En general, M. mola presenta una distribución cosmopolita, aunque preferentemente en aguas cálidas, donde efectúa movimientos estacionales horizontales nadando a través corrientes marinas y permaneciendo en determinadas áreas para su alimentación, aunque son capaces de moverse a grandes distancias y bajar hasta 600-800 metros de profundidad en búsqueda de presas potenciales (Cartamil \& Lowe 2004; Sims et al. 2009a, 2009b). En aguas chilenas su presencia había sido documentada previamente solo en dos ocasiones en Chile central (Kong et al. 1985; Brito 2003), y

TABLA 2. Lista taxonómica de las especies de parásitos registrados en peces de la familia Molidae en aguas de Chile, incluyendo los reportes de este estudio. Se detalla taxa parasitarios, hospedero, lugar del hallazgo y autor del trabajo.

TABLE 2. Taxonomic list of species of fish parasites registered in the Molidae family in Chilean waters, including reports of this study. Parasitic taxa, host, instead of finding and author of the work are detailed.

\begin{tabular}{|c|c|c|c|}
\hline Taxa parasitario & HoSPEDERO & LUGAR (coordenadas) & AutOR/ES \\
\hline \multicolumn{4}{|l|}{ MONOGENEA } \\
\hline \multirow[t]{2}{*}{ Capsala martinieri Bosc, 1811} & M. ramsayi & Golfo de Arauco ( $\left.37^{\circ} 00^{\prime} \mathrm{S}\right)$ & Villalba \& Fernández (1985) \\
\hline & M. ramsayi & Las Cruces $\left(33^{\circ} 31^{\prime} \mathrm{S}\right)$ & Brito (2003) \\
\hline \multicolumn{4}{|l|}{ DIGENEA } \\
\hline Accacladium serpentulum Odhner, 1928 & M. ramsayi & Isla de Pascua $\left(27^{\circ} \mathrm{S} 109^{\circ} \mathrm{O}\right)$ & Villalba \& Fernández (1985) \\
\hline \multirow{2}{*}{ Accacladocoelium macrocotyle (Diesing, 1858) } & M. mola & Tomé $\left(36^{\circ} 37^{\prime} \mathrm{S}\right)$ & Presente estudio \\
\hline & M. ramsayi & Isla de Pascua $\left(27^{\circ} \mathrm{S} 109^{\circ} \mathrm{O}\right)$ & Villalba \& Fernández (1985) \\
\hline \multirow{3}{*}{ Accacoelium contortum (Rudolphi, 1819) } & M. ramsayi & Golfo de Arauco ( $\left.37^{\circ} 00^{\prime} \mathrm{S}\right)$ & Villalba \& Fernández (1985) \\
\hline & M. ramsayi & Isla de Pascua $\left(27^{\circ} \mathrm{S} 109^{\circ} \mathrm{O}\right)$ & Villalba \& Fernández (1985) \\
\hline & M. mola & Tomé (36³7’s) & Presente estudio \\
\hline Odhnerium calyptrocotyle (Monticelli, 1893) & M. ramsayi & Isla de Pascua $\left(27^{\circ} \mathrm{S} 109^{\circ} \mathrm{O}\right)$ & Villalba \& Fernández (1985) \\
\hline \multicolumn{4}{|l|}{ NEMATODA } \\
\hline Anisakis sp. Tipo I sensu Berland, 1961 (L3) & M. mola & Tomé ( $\left.36^{\circ} 37^{\prime} S\right)$ & Presente estudio \\
\hline \multicolumn{4}{|l|}{ CESTODA } \\
\hline \multirow{2}{*}{ Anchistrocephalus microcephalus (Rudolphi, 1819) } & M. ramsayi & Golfo de Arauco ( $\left.37^{\circ} 00^{\prime} \mathrm{S}\right)$ & Villalba \& Fernández (1985) \\
\hline & M. mola & Tomé $\left(36^{\circ} 37^{\prime} \mathrm{S}\right)$ & Presente estudio \\
\hline \multirow[t]{2}{*}{ Molicola horridus (Goodsir, 1841) (plerocerci) } & M. ramsayi & Isla de Pascua $\left(27^{\circ} \mathrm{S} 109^{\circ} \mathrm{O}\right)$ & Villalba \& Fernández (1985) \\
\hline & M. mola & Tomé (36³7’S) & Presente estudio \\
\hline Nybelinia spp. (plerocerci) & M. ramsayi & Isla de Pascua $\left(27^{\circ} \mathrm{S} 109^{\circ} \mathrm{O}\right)$ & Villalba \& Fernández (1985) \\
\hline No determinados & M. ramsayi & San Antonio $\left(33^{\circ} 35^{\prime} \mathrm{S}\right)$ & Brito (2003) \\
\hline \multicolumn{4}{|l|}{ COPEPODA } \\
\hline Pennella cf. filosa Linneo, 1758 & M. ramsayi & Zapallar (3331'S) & Atria (1967) \\
\hline \multirow[t]{2}{*}{ Cecrops latreilli Leach, 1816} & M. ramsayi & Isla de Pascua $\left(27^{\circ} \mathrm{S} 109^{\circ} \mathrm{O}\right)$ & Villalba \& Fernández (1985) \\
\hline & M. mola & Tomé $\left(36^{\circ} 37^{\prime} \mathrm{S}\right)$ & Presente estudio \\
\hline \multirow[t]{2}{*}{ Lepeophtheirus nordmanni (Milne Edwards, 1840) } & M. ramsayi & Golfo de Arauco ( $\left.37^{\circ} 00^{\prime} \mathrm{S}\right)$ & Villalba \& Fernández (1985) \\
\hline & M. mola & Valparaíso $\left(33^{\circ} 02^{\prime} \mathrm{S}\right)$ & Stuardo (1958) \\
\hline
\end{tabular}


estarían relacionadas a la presencia del evento de El Niño. De esta forma, este nuevo registro establece su ubicación más austral en Tomé, en el litoral centro sur de Chile.

Cabe destacar que todos los parásitos aislados desde el ejemplar del presente estudio, ya habían sido registrados previamente en peces $M$. mola de distintas ubicaciones geográficas. Sin embargo, el nematodo Anisakis sp. Tipo I, no había sido reportado en $M$. mola en aguas chilenas (Tabla 2). Por otro lado, se han informado algunas diferencias en la conformación de la parasitofauna de M. mola y distintas tasas de intensidad de infección, lo que sería el reflejo ontogénico de estas poblaciones en particular. Dentro de este contexto, todos los parásitos reportados en el presente estudio son transmitidos al hospedero por el consumo de presas infectadas, excepto el copépodo C. latreilli que se transmite por contacto directo. De este modo, las variaciones cuantitativas y cualitativas de estos taxa parásitos dependen de la tasa de encuentros entre el depredador y presas parasitadas, es decir, de la composición de su dieta (Campbell et al. 1980; Rohde 2005). En general, la dieta del pez luna se basa principalmente en presas gelatinosas del zooplancton (Cardona et al. 2012; Nakamura \& Sato 2014), aunque se ha mencionado además la posibilidad de alimentación a partir de medusas, ctenóforos, salpas, crustáceos, ofiuros, moluscos, algas, peces (Fraser-Brunner 1951; Hart 1973; Scott \& Scott 1988) y descartes de pesca (Syväranta et al. 2012).

Los resultados ratifican la participación de $M$. mola como hospedero definitivo, intermediario o paraténico en los ciclos de vida de los parásitos identificados en este estudio, los cuales son, excepto Anisakis sp. Tipo I, especialistas de mólidos. Si bien estos ciclos no se conocen completamente, Grabda (1991) y Ahuir-Baraja (2012) señalan que pequeños crustáceos, medusas y quetognatos actuarían como hospederos intermediarios para los digeneos, nematodos y cestodos encontrados en peces luna. De la misma forma, los depredadores de $M$. mola serían los hospederos definitivos de los estados larvales encontrados en este estudio. Por ejemplo, las larvas plerocerci de $M$. horridus se convertirían en estados adultos en elasmobranquios, reconocidos depredadores de M. mola (Pope et al. 2010) mientras que las larvas de Anisakis sp. Tipo I completarían su ciclo en cetáceos como las orcas (Fergusson et al. 2000; Ryan \& Holmes 2012). La presencia de larvas de Anisakis sp. Tipo I en este hospedero había sido descrita por primera vez recientemente en ejemplares del Mediterráneo (AhuirBaraja, 2012). Su hallazgo en el ejemplar analizado, el primero en mólidos en aguas chilenas, no es casual ya que este nematodo es un parásito generalista descrito en numerosos hospederos que habitan el litoral de Chile (Torres et al. 1983). De la misma forma, la presencia del copépodo $C$. latreilli, ha sido ampliamente registrada en
M. mola de diversas localizaciones (Gustinelli et al. 2006; Ahuir-Baraja, 2012) debido a su elevada especificidad por hospederos mólidos. Sin embargo, llama la atención su baja intensidad de infección en el pez analizado, en este estudio, en comparación a reportes anteriores (Díaz 2000) y la ausencia del copépodo Lepeophtheirus nordmannii, cuya detección es frecuente en estas especies. No obstante, esta situación no implica necesariamente la ausencia y/o baja carga parasitaria de estas especies ya que, una vez muerto el ejemplar en cuestión, eventuales ectoparásitos pudieron haberse desprendido de las estructuras que le servían de sustrato.

Como se mencionó anteriormente, la observación de ejemplares de Molidae en las aguas del litoral centro sur de Chile es inusual y podría ser resultado de eventos de naturaleza climática (Sielfeld et al. 2010). Sin embargo, y a la luz de los resultados expuestos, que evidencian una alta intensidad de infección parasitaria en el ejemplar analizado, cabe plantear interrogantes respecto de cuál es el papel que juegan los parásitos como factores predisponentes o determinantes en los fenómenos de varamiento de estas especies ya que, podría estar relacionado con la alteración de las condiciones de bienestar fisiológico del hospedero eventual (Iwanowicz 2011). Por ejemplo, se reconoce que las larvas plerocerci de $M$. horridus podrían estar relacionados al deterioro funcional de hígado y riñones. No obstante, Ahuir-Baraja (2012) correlacionó diferentes estimadores de la carga del parásito (intensidad, biomasa, densidad y porcentaje de superficie hepática ocupada) con índices de condición de salud del pez (Índice Hepatosomático y Factor de Condición), señalando que se necesita una pérdida considerable de tejido funcional para que este órgano parezca notablemente afectado. Por su parte, la ocurrencia de alta carga de parásitos como $C$. latreillii en branquias y mucosas incrementaría la susceptibilidad a infecciones de carácter secundario, que podrían determinar debilitamiento y muerte del hospedero (Díaz 2000). Por último, cargas elevadas de céstodos intestinales, como A. microcephalus, se podrían asociar a retraso en desarrollo y crecimiento, además de cuadros carenciales debido a la acción expoliatriz que efectuarían en la mucosa intestinal (Möller \& Anders 1986). Debido a la escasez de ejemplares y la carencia de evaluaciones, desde el punto de vista histopatológico en la literatura, no es posible dilucidar estos fenómenos.

A modo de conclusión, consideramos necesario el proseguir con estudios respecto del parasitismo en $M$. mola $\mathrm{y}$, en general, en todos los mólidos dado que la identificación de estas especies de parásitos y su interacción patogénica con su hospedero, podrían aportan información respecto de la biología, historia de vida y aspectos de salud animal, que permitan aclarar cuál es el posible rol de los parásitos en los fenómenos de varamiento de estas especies. 


\section{AGRADECIMIENTOS}

Este trabajo fue financiado por el proyecto de la Universidad de Concepción, VRID N² 213.036.040-1.0.

\section{BIBLIOGRAFÍA}

Ahuir-Baraja, A.E. 2012. Estudio parasitológico del pez luna Mola mola (L.), en el Mediterráneo Occidental. Tesis Doctoral. Facultat de Ciéncies Biológiques, Universitat de Valencia, Valencia, España. 343 pp.

AtriA, G. 1967. Un ectoparásito del pez luna (Mola ramsayi Giglioli) Pennella cf. filosa L. (Crustacea, Copepoda). Noticiero Mensual del Museo Nacional de Historia Natural, Chile, 131:3-5.

AtriA, G. 1977. Lista de copépodos asociados a organismos marinos en Chile (Caligoida, Lernaeopoida y Cyclopoida). Noticiero Mensual del Museo Nacional de Historia Natural, Chile, 21(247-248):3-7.

Bass, A.L., H. Dewar, T. Thys, J. Streelman \& S. Karl. 2005. Evolutionary divergence among lineages of the ocean sunfish family, Molidae (Tetraodontiforms). Marine Biology, 148 (2):405-414.

Berland, B. 1961. Nematodes from some Norwegian marine fishes. Sarsia, 2: 1-50.

Bray, R. \& I. Gibson. 1977. The Accacoelidae (Digenea) of fishes from the north-east Atlantic. Bulletin of the British Museum. (Natural History), 31 (2): 53-99.

Bray, R., A. Jones \& K. Anderdsen. 1994. Order Pseudophyllidea Carus, 1863. In: L.F. Khalil, A. Jones, R.A., Bray (eds.), Keys to the Cestode Parasites of Vertebrates. Cab International, 10: 205-246.

BRITO, J. 2003. Nuevos registros de Balistes polylepis (Balistidae), Sphoeroides lobatus (Tetraodontidae), Mola mola y M. ramsayi (Molidae) en San Antonio, Chile (Pisces, Tetraodontiformes). Investigaciones Marinas, Valparaíso, 31(1): 77-83.

Campbell, R., R. Haedrich \& T. Munro. 1980. Parasitism and ecological relationships among deep-sea benthic fishes. Marine Biology, 57: 301-313.

Cardona, L., I. Álvarez De Quevedo, A. Borrell \& A. Aguilar. 2012. Massive consumption of gelatinous plankton by Mediterranean apex predators. Plos One 7(3): 1-14.

Cartamil, D.P. \& C.G. Lowe. 2004. Fine-scale movement patterns of ocean sunfish (Mola mola) off southern California. Marine Ecology Progress Series, 266: 245-253.

DíAz, O. 2000. Copépodos ectoparásitos del pez luna Mola mola (Giglioli, 1883) (Pisces: Molidae) en el Golfo de Cariaco, Venezuela. Boletín del Instituto Oceanográfico, Venezuela, Universidad. Oriente 39 (1-2): 11-7.

Fergusson, I., L. Compagno \& M. Marks. 2000. Predation by white sharks Carcharodon carcharias (Chondrichthyes: Lamnidae) upon chelonians, with new records from the Mediterranean Sea and a first record of the ocean sunfish Mola mola (Osteichthyes: Molidae) as stomach contents. Environmental Biology of Fishes, 58: 447-453.

Fraser-BrunNer, A. 1951. The Ocean sunfishes (Family Molidae). Bulletin of the British Museum. (Natural History), 1(6):
89-121.

Grabda, J. 1973. Contribution to knowledge of biology of Cecrops latreilli Leach, 1816 (Caligoida: Cecropidae) the parasite of the ocean sunfish Mola mola (L.) Acta Ichthyologica et Piscatoria, 3 (1): 61-72.

Grabda, J. 1991. Marine Fish Parasitology: An Outline. Polish Scientific Publishers, Warszawa. Poland. 306 pp.

Gustinelli, A., G. Nardini, G. Aureli, M. Trentini, M. Affronte \& M. Fioravanti. 2006. Parassitofauna di Mola mola (Linnaeus, 1758) dei mari italiani. Biologia Marina Mediterranea, 13(1): 872-876.

Hart, J.L. 1973. Pacific Fishes of Canada. Fisheries Research Board of Canada Bull., 180: 740 pp.

Houghton, J.D., T. Doyle, J. Davenport \& G. Hays. 2006. The ocean sunfish Mola mola: insights into distribution, abundance and behavior in the Irish and Celtic Seas. Journal of the Marine Biological Association Of the United Kingdom, 86: 1-7.

IwANOWICZ, D.D. 2011. Overview on the effects of parasites on fish health. In: Bridging America and Russia with Shared Perspectives on Aquatic Animal Health (Eds. Cipriano R.C. A.W. Bruckner, \& I.S. Shchelkunov), pp. 176-184. Proceedings of the Third Bilateral Conference between Russia and the United States, 12-20 July, 2009, held in Shepherdstown, West Virginia. Landover, Maryland, USA.

Kong, I., J. Tomicic \& J. Zegers. 1985. Ictiofauna asociada al fenómeno de El Niño 1982-83 en la zona norte de Chile. Investigaciones Pesqueras, Chile, 32: 215-224.

Möller, H. \& K. Anders. 1986. Diseases and Parasites of Marine fishes. Verlag Möller. Kiel, Germany. 365 pp.

MuÑoz, G. \& V. Olmos. 2007. Revisión bibliográfica de especies ectoparásitas y hospedadoras de sistemas acuáticos de Chile. Revista de Biología Marina y Oceanografía, 42(2): 89-148.

NaKamura, I. \& K. Sato. 2014. Ontogenetic shift in foraging habit of ocean sunfish Mola mola from dietary and behavioral studies. Marine Biology, 161 (6), 1263-1273.

Parenti, P. 2003. Molidae Bonaparte 1832, molas or ocean sunfishes. In: Annotated Checklist of fishes $\mathrm{N}^{\circ} 18$, California Academy of Sciences, 9 pp.

Pequeño, G. 1989. Peces de Chile. Lista sistemática revisada y comentada. Revista de Biología Marina, Valparaíso, 24 (2): $1-132$

Pequeño, G. 1997. Peces de Chile. Lista sistemática revisada y comentada: addendum. Revista de Biología Marina y Oceanografía, 32 (2): 77-94.

Pope, E.C., G. Hays, T. Thys, T. Doyle, D. Sims, N. Queiroz, V. Hobson, L. Kubicek \& J. Houghton. 2010. The biology and ecology of the ocean sunfish Mola mola: a review of current knowledge and future research perspectives. Reviews of Fish Biology and Fisheries, 20: 471-487.

Rohde, K. 2005. Marine Parasitology. CABI publishing, Wallingford. United Kingdom, 565 pp.

Ryan, C. \& J. Holmes. 2012. Killer whale Orcinus orca predation on sunfish Mola mola. Marine Biodiversity Records, 672: 1100-1187.

Scott, W.B. \& M.G. Scott. 1988. Atlantic fishes of Canada. Canadian Bulletin of Fish Aquatic Science, 219: 731 pp.

Sielfeld, W., J. Laudien, M. Vargas \& M. Villegas. 2010. El Niño induced changes of the Coastal fish fauna off northern 
Parásitos de Mola mola en la costa centro-sur de Chile: ItAlo FernÁndez ET AL.

Chile and implications for ichthyogeography. Revista de Biología Marina y Oceanografía, 45 (1): 705-722.

Sims, D.W., N. Queiroz, T. Doyle, J. Houghton \& G.C. Hays. 2009a. Satellite tracking of the world's largest bony fish, the ocean sunfish (Mola mola) in the North East Atlantic. Journal of Experimental Marine Biology and Ecology, 370: 127-133.

Sims, D.W., N. QueIroz, N.E. Humphries, F.P. Lima \& G.C. HaYs. 2009b. Long-term GPS tracking of ocean sunfish Mola mola offers a new direction in fish monitoring. PLoS ONE, 4: e7351.

Stuardo, J. 1958. Lepeophtheirus ornatus (Milne Edwards) a synonym of L. nordmanni (Milne Edwards) (Copepoda: Caligidae) Univ. Bergen Arbok Naturvitensk Rekke 8: $1-11$.

Syväranta, J., C. Harrods, L: Kubicek, V. Cappanera \& J. Houghton. 2012. Stable isotopes challenge the perception of ocean sunfish Mola mola as obligate jellyfish predators. Journal of Fish Biology, 80: 225-231.
Torres, P., E. Hernández \& I. Sandoval. 1983. Anisakiasis and phocanemiasis in marine fishes from south of Chile. International Journal of Zoonoses, 10: 146-150.

Villalba, C. \& J. Fernández. 1985. Parásitos de Mola ramsayi (Giglioli, 1883) (Pisces: Molidae) en Chile. Boletín de la Sociedad de Biología de Concepción, Chile, 56: 71-78.

Yamaguti, S. 1961. Systema Helminthum: The Nematodes of Vertebrates. Volume III. Interscience Publs. New York. USA. 679 pp.

Yoshita, Y., Y. Yamanoue, K. Sagara, M. Nishibori, H. Kuniyoshi, T. Umino, Y. Sakai, H. Hashimoto \& K. Gushima. 2009. Phylogenetic relationship of two Mola sunfishes (Tetraodontiformes: Molidae) occurring around the coast of Japan, with notes on their geographical distribution and morphological characteristics. Ichthyological Research, 56 (3): 232-244.

Recibido: 16.06 .16

Aceptado: 12.12 .16 